\title{
Prévalence Et Diversité De Salmonella En Afrique : Analyse Qualitative Et Quantitative
}

\author{
Alio Sanda Abdelkader \\ Université Abdou Moumouni, Faculté des Sciences et Techniques, \\ Département de Biologie, Laboratoire Garba Mounkaila et Laboratoire de \\ Gestion et Valorisation de la Biodiversité au Sahel, Niamey (NIGER) \\ Samna Soumana Oumarou \\ Université de Tillabéri, Faculté des Sciences Agronomiques et de \\ l'Environnement. Tillabéri, (NIGER)

\section{Inoussa Maman Maârouhi Diallo Bouli Ali Bakasso Yacoubou} \\ Université Abdou Moumouni, Faculté des Sciences et Techniques, \\ Département de Biologie, Laboratoire Garba Mounkaila et Laboratoire de \\ Gestion et Valorisation de la Biodiversité au Sahel, Niamey (NIGER)
}

Doi: 10.19044/esj.2017.v13n30p250 URL:http://dx.doi.org/10.19044/esj.2017.v13n30p250

\begin{abstract}
International trade, new agricultural, animal production and food practices have facilitated the spread and transmission of food-borne pathogens; including Salmonella. Salmonella is a ubiquitous bacterium responsible for a variety of diseases in humans, animals. It has a large diversity and contains more than 2579 serotypes. However, very little work describing the diversity, structure and populations dynamics of Salmonella in different hosts is available in Niger. In order to describe the general context of Salmonella diversity in Africa, an analysis of the available literature on this subject was carried out. A total of 131 publications were identified. From those publications, twenty five (25) were eligible. The LOGIT estimation model was used for serotype analysis to standardize the distribution of data and minimize sample sizes. It appears from our analysis that the prevalence of Salmonella is high regardless of the host and host germ. The major serotypes found in humans in Africa are $S$. Typhimurium, $S$. Enteritidis and S. Typhi. In poultry, the predominant serotype is S. Hadar on the other side S. Typhimurium predominates in North Africa. Thus, the results of these review analyzes constitute one of the steps in the process of understanding trends in the distribution of Salmonella in Africa. These results are presented as an outline of the implementation of a successful
\end{abstract}


model for the continuation of our studies on the diversity of Salmonella in Niger.

Keywords: Salmonella, serotypes, diversity, Niger, Africa

\section{Résumé}

Les échanges internationaux, les nouvelles pratiques agricoles, de production animale et d'alimentation, ont facilité la propagation et la transmission d'agents pathogènes d'origine alimentaire; notamment Salmonella. Salmonella est une bactérie ubiquiste responsable de pathologies diverses chez l'homme, les animaux. Elle présente une forte diversité avec à ce jour plus de 2579 sérotypes connus. Cependant, très peu des travaux décrivant la diversité, la structuration et le dynamisme des populations de Salmonella dans différents hôtes sont disponibles au Niger. Pour décrire le contexte général de la diversité de Salmonella en Afrique, une analyse des revues disponibles traitant de ce sujet a été effectuée. Cent trente-et-une (131) publications ont été recensées. Dans cette gamme de publications, vingt-cinq (25) ont été retenues après application d'un certain nombre de critères de sélection. Le modèle d'estimation de LOGIT a été utilisé pour l'analyse des sérotypes afin de normaliser la distribution des données et minimiser les tailles des échantillons. Il ressort de notre analyse que la prévalence des Salmonella est élevée quelque soit 1'hôte.Le germe présente une grande diversité d'un hôte à l'autre. Les sérotypes majeurs retrouvés chez l'homme en Afrique sont $S$. Typhimurium, $S$. Enteritidis et $S$. Typhi. Chez la volaille, le sérotype prédominant est $S$. Hadar sauf en Afrique du Nord, où c'est $S$. Typhimurium qui prédomine. Les résultats de ces analyses de revues constituent une étape de la comprehension de la distribution des Salmonella en Afrique un point important pour nos études sur la diversité de Salmonella au Niger.

Mots clés : Salmonella, sérotypes, diversité, Niger, Afrique

\section{Introduction}

Les salmonelloses sont les principales causes des toxi-infections alimentaires collectives chez l'homme. On estime à 93,8 millions cas de gastro-entérites $\left(5^{\mathrm{e}}-95^{\mathrm{e}}\right.$ percentile, 61,8-131,6 millions) dues aux Salmonella. On dénombre 155.000 décès, parmi eux 80,3 millions sont d'origine alimentaire (Shannon et al., 2010). En pathologie humaine, les Salmonella sont divisées en sérotypes typhiques ( $S$. Typhi et $\mathrm{S}$. Paratyphi) et Sérotypes Non Typhiques. Contrairement au pays développés, en Afrique subsaharienne, les Salmonella non Typhoïques (NTS) sont identifiées comme les principales bactéries isolées dans le sang chez les 
adultes et les enfants et sont associées à 20 à $25 \%$ de décès. La détermination des sérotypes est gouvernée par une mosaïque de gènes recombinant entre eux et codant pour les antigènes capsulaires de surface. Ces mécanismes confèrent à la bactérie sa forte diversité mais aussi les moyens de s'adapter à la réponse immunitaire et aux antibiotiques. En effet, la dynamique de la diversité des Salmonella est fonction des régions géographiques et des hôtes (Coulibaly et al., 2010). Avec l'arrivée des outils de sérotypage, s'est développé une meilleure surveillance des épidémies des Salmonella et la détection de leurs sources, leur suivi dans le temps et l'attribution de la source de contamination. Malgré cela, aujourd'hui, la distribution globale des sérotypes de Salmonella humaines est très peu connue.

Depuis Janvier 2000, l'Organisation Mondiale de la Santé (OMS) a lancé le Réseau mondial des infections d'origine alimentaire (GFN) (connu sous le nom du Programme mondial Salm-Surv (GSS)).Un effort mondial visant à renforcer la surveillance des infections et d'autres maladies d'origine alimentaire en laboratoire, et de promouvoir des activités de prévention et de contrôle. Ainsi les résultats montrent que $S$. Typhimurium et $S$. Enteritidis sont largement répandues dans les différents réservoirs animaux (porcs, bovins, volailles, ...), dans certaines denrées alimentaires (œufs, fruits, ...) destinées à l'homme et figurent en première et deuxième position des sérotypes les plus fréquemment isolés chez l'homme (Hendriksen, 2010) (GFN) http://www.who.int/gfn/en/. La plupart des cas de Salmonella non Typhoïques isolées en Afrique sont des $S$. Typhimurium ou $S$. Enteritidis, (Nicholas et al., 2012) bien que d'autres investigations rapportent la contribution d'autres sérotypes $S$. Isangi en Afrique du Sud (Wadula et al., 2009), S. Concord en Ethiopie (Beyene et al., 2011), et S. Dublin au Mali (Tennant et al., 2010). La distribution globale des sérotypes chez l'homme est complexe, du fait de l'influence des facteurs tels que les réservoirs animaux et/ou environnementaux et la complexité des voies de transmission. $\mathrm{Au}$ regard de tout ce qui précède, il est important d'étendre l'étude de la diversité à tous les réservoirs

L'objectif est de faire une revue des données disponibles de la diversité des Salmonella en Afrique. Afin de fournir une mise à jour sur la diversité génétique, et la distribution du genre Salmonella en fonction des hôtes et des régions géographiques.

\section{Méthodologie}

\section{Identification des articles}

La recherche bibliographique est effectuée dans le moteur de recherche «Google» et dans «Pubmed». Le but est d'identifier la prévalence, la distribution, la diversité et l'épidemiologie des Salmonella en 
Afrique. Les termes utilisés dans le moteur de recherche sont: «Salmonella » en combinaison avec le pays concerné.

\section{Eligibilité des études}

Une étude est éligible pour l'analyse si :

- $\quad$ Son objectif n'est pas d'étudier un sérotype spécifique,

- $\quad$ La taille de la population totale étudiée est bien décrite,

- $\quad$ La taille des échantillons positifs de Samonella est bien décrite,

- $\quad$ Les différents sérotypes de Salmonella trouvés sont bien décrits.

\section{Sélection des articles}

Les articles dont seuls les titres sont obtenus ont été exclus de l'analyse, de même que les résumés et les textes complets dont les auteurs n'ont travaillé que sur des sérotypes spécifiques. Les résumés et les textes complets qui ont été receuillis plusieurs fois été exclus [Gedda,. (2015)].

\section{Extraction des données}

Pour toute étude retenue, les données suivantes sont collectées et notées dans un tableau Excel. Il s'agit: du premier auteur, du titre de l'article, du titre du journal, de l'espèce étudiée, de l'hôte étudié, du pays où l'étude à été realisée, du site de prélèvement, de la taille de la population totale, de la taille des échantillons positifs, des techniques de sérotypage, et des sérotypes.

\section{Analyse des données} formule :

La prévalence des échantillons (p) est calculée suivant la

$$
\mathbf{P}=\frac{\mathbf{n}(\text { échantillons positifs) }}{\mathbf{N}(\text { échantillons totals })}
$$

- $\quad$ L'erreur standard (SE) suivant la formule: $\mathbf{S E}=\frac{\mathbf{P}}{\sqrt{\mathbf{P N}}}$, avec $\mathbf{n}=$ le nombre d'échantillons positifs, $\mathbf{N}=$ le nombre total d'échantillons.

- $\quad$ La variance est calculée suivant la formule : $\mathbf{V a r}=\mathbf{S E}^{2}$.

Afin de normaliser la distribution des données et d'integrer l'influence du poids des échantillons sur le résultat, le poids individuel des études (w) est calculé.

- $\quad$ Le poids de chaque étude est égal avec l'inverse de la variance.

Ainsi $\mathbf{w}=\frac{1}{\mathbf{S E}^{2}}$

Le poids des effets ponderés est déduit de la formule ( $\left.\mathbf{w}^{*} \mathbf{P}\right)$ (Tadesse, 2014a; Tadesse et al., 2014b).

\section{Hétérogénéité et biais de l'analyse.}


Les différentes techniques d'isolements et d'identifications des Salmonella ainsi que les droites de régressions sont utlisées pour évaluer visuellement les biais des études. Le rôle biaisant des types d'études a été évalué en utilisant un coefficient de corrélation $\mathrm{R}^{2}$ et l'intercept. Le niveau de significativité a été établi a` 0,05 .

Pour évaluer la variabilité de l'effet du traitement selon les études, on utilise le test d'hétérogénéité dit de Cochran [Cochran WG., 1954] . Sous l'hypothèse nulle de l'homogénéité de l'effet du traitement entre les $\mathbf{n}$ études, la statistique $\mathbf{Q}$ ci-après suit une loi de $\mathrm{Chi}^{2}$ à $\mathrm{n}-1$ degrés de liberté :

$$
\mathbf{Q}=\sum\left(\mathbf{w} \mathbf{P}^{-2}\right)-\frac{\left[\sum \mathbf{w P}\right]}{\sum \mathbf{w}},
$$

Les taux de variation attribués à l'hétérogénéité sont quantifiés par le calcul de :

$$
\mathbf{I}^{2}=\frac{(\mathbf{Q}-\mathbf{d f})}{\mathbf{Q}} \mathbf{1 0 0} \text {. }
$$

Les études sont considérées comme hétérogènes lorsque le rapport entre $\mathbf{Q}$ et le degré de liberté $\mathbf{d f}$ est supérieur à $1 . \mathrm{I}^{2}$ ne dépend pas du nombre d'études considérées.

- $\quad \quad \quad \quad$ Si $\mathrm{I}^{2}<0, \mathrm{I}^{2}$ est considéré comme nul.

- $\quad$ Si $\mathrm{I}^{2}<25 \%$ cela indique une hétérogénéité faible,

- $\quad$ Si $25<\mathrm{I}^{2}<75 \%$ on a une hétérogénéité modérée

- $\quad \mathrm{I}^{2}>75 \%$ on parle d'hétérogénéité importante.

Les analyses ont été réalisées sur les logiciels Excel et MedCaL. 


\section{Résultats}

\section{Recherche de la littérature et éligibilité des études}
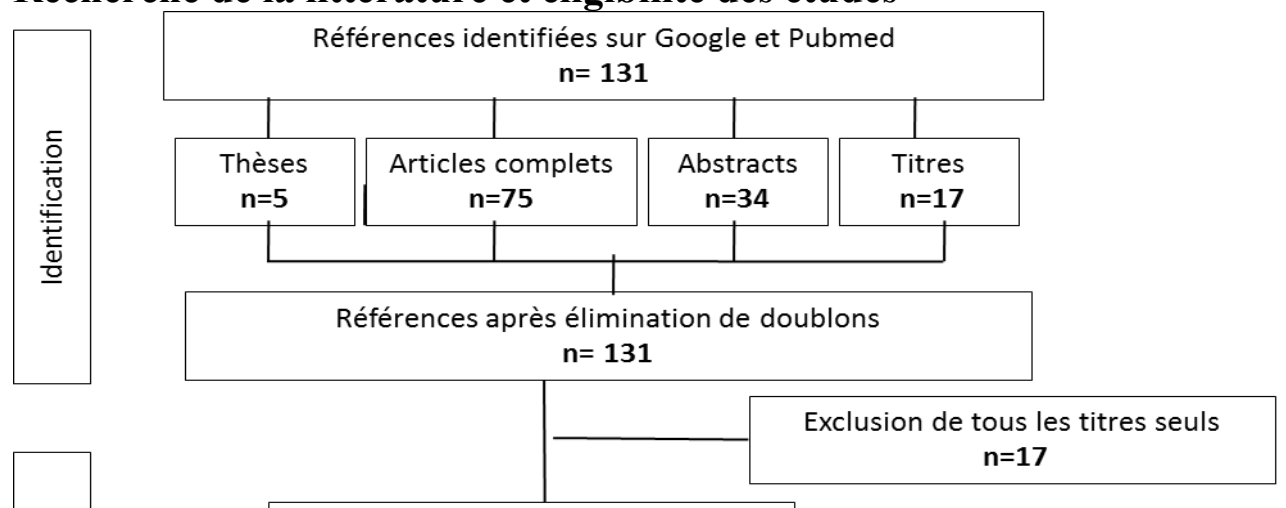

Références sélectionnées

(Articles complets et résumés)

$n=114$

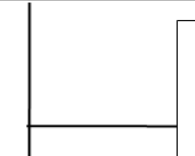

Références exclues car études réalisées sur des sérotypes spécifique et des Salmonella non typhoïdiques $\mathrm{n}=\mathbf{3 5}$

Références exclues car études réalisées sur les aliments et/ou l'environnement $n=11$

\section{Références retenues pour la synthèse} $\mathrm{n}=\mathbf{2 5}$

Figure 1 : Diagramme de sélection des études éligilibles.

La figure 1 présente les résultats de la recherche des articles. Cette recherche a permis de trouver cent trente-et-une (131) études. Dix-sept (17) études présentant seulement des titres ont été immédiatement exclues. Sur les cent-quatroze (114) rétenues, trente-cinq (35) dont les auteurs ont travaillé 
sur des sérotypes spécifiques, de même trente-sept (37) ayant des données manquantes sur l'échantillon total et/ou le sérotype, ont été exclues. Six (6) études rétrospectives ont été exclues. De plus onze (11) autres études ont été exclues, parcequ'elles ont été faites exclusivement sur des aliments et/ou sur l'environemment. Au total seules vingt-cinq (25) études sont donc retenues.

\section{Risque de biais des publication}

Tableau 1 : Techniques utilisées pour l'isolement des Salmonella.

\begin{tabular}{|c|c|c|c|c|}
\hline Hôtes & Auteurs/Pays/Dates & $\begin{array}{c}\text { Pré- } \\
\text { enrichissemen } \\
t\end{array}$ & $\begin{array}{c}\text { Enrichisseme } \\
\text { nt }\end{array}$ & Isolement \\
\hline \multirow{6}{*}{$\begin{array}{c}\text { Humai } \\
\mathrm{n}\end{array}$} & $\begin{array}{c}\text { Le Noc [Cameroun } \\
1976]\end{array}$ & $\mathrm{nr}$ & GN & XLD \\
\hline & Ammari [Maroc 2009] & BPW & RVS & Hecktoen \\
\hline & Fashae [Nigeria 2010] & $\mathrm{BHI}$ & SF & DCA, XLD \\
\hline & Beyene [Ethiopie 2011] & BHI & $\mathrm{nr}$ & SS, McContey \\
\hline & Dembélé [Burkina 2014] & BHI & $\mathrm{nr}$ & SS, McContey \\
\hline & Langendorf [Niger 2015] & $\begin{array}{l}\text { CaryBlair } \\
\text { Tube }\end{array}$ & $\mathrm{nr}$ & Hecktoen \\
\hline \multirow{11}{*}{$\begin{array}{c}\text { Volaill } \\
\mathrm{e}\end{array}$} & Uche Orji [Nigeria 2005] & $\mathrm{nr}$ & SF & DCA \\
\hline & Chaiba [Maroc 2009] * & BPW & SC, RVS & SS \\
\hline & Ammar [Algérie 2010] * & BPW & RVS, SC & BGA, Hecktoen \\
\hline & $\begin{array}{c}\text { El Hussein [Soudan } \\
2010] ¥\end{array}$ & \multicolumn{3}{|c|}{ ISO6579 (1998) } \\
\hline & Ashraf [Egypte 2012] & $\mathrm{nr}$ & RVS & XLD \\
\hline & Boko [Bénin 2012] & BPW & RVS & XLD, SS \\
\hline & $\begin{array}{l}\text { Bounar-Kechih [Algérie } \\
\text { 2012]! }\end{array}$ & \multicolumn{3}{|c|}{ NF U47-100/2007 } \\
\hline & Mezali [Algérie 2012] ! & \multicolumn{3}{|c|}{ NF V08-052 } \\
\hline & $\begin{array}{c}\text { Karou [Côte d'Ivoire } \\
\text { 2013] } * ¥\end{array}$ & BPW & RVS, MKTT & $\begin{array}{c}\text { Hecktoen, SS } \\
\text { (ISO6579 (2002) }\end{array}$ \\
\hline & $\begin{array}{c}\text { El Allaoui [Maroc 2014] } \\
!\end{array}$ & \multicolumn{3}{|c|}{ NF V08-052 } \\
\hline & $\begin{array}{c}\text { Kagambèga [Burkina } \\
\text { Faso 2013] }\end{array}$ & BPW & RVS & XLD \\
\hline \multirow{7}{*}{ Bétail } & Uche Orji [Nigeria 2005] & $\mathrm{nr}$ & $\mathrm{SF}$ & DCA, PDGA \\
\hline & Stevens [Sénégal 2006] * & BPW & SC, RVS & Hecktoen \\
\hline & $\begin{array}{c}\text { Kagambèga [Burkina } \\
\text { Faso 2010] }\end{array}$ & BPW & $\begin{array}{l}\text { SC, RVS, } \\
\text { MKTTn }\end{array}$ & XLD \\
\hline & Kikuvi [Kenya 2010] & \multicolumn{3}{|c|}{ BPW } \\
\hline & Mezali [Algérie 2012] & BPW & Tétrathionate & DCA \\
\hline & $\begin{array}{c}\text { Abbassi-Ghozzi [Tunisie } \\
\text { 2012] * }\end{array}$ & BPW & SC, RVS & XLD, Hecktoen, \\
\hline & $\begin{array}{c}\text { Shilangale [Namibie } \\
\text { 2012]* }\end{array}$ & BPW & SC, RVS & BS, Hecktoen \\
\hline Végéta & Ndiaye [Sénégal2011] & $\mathrm{nr}$ & RVS & XLD \\
\hline
\end{tabular}


ux

Abakpa [Nigeria 2013]

Bagudo [Nigeria 2014]

$\mathrm{nr}$

RVS

XLD

Traoré [Burkina 2015]

$\mathrm{nr}$

SF

SS

BPW

RVS

Hecktoen

BHI : Bouillon Cœur Cervelle ; BPW : Bouillon Eau Peptonée ; DCA : Gélose Deoxycholate Citrate ; GN : Gélose Nutritive; PDGA : Gélose Propylène Glycol Deoxycholate ; RVS : Bouillon Rappaport-Vassiliadis ; SC : Sélénite-Cystine ; SF :

Bouillon Sélénite-F; SS : Gélose Salmonella Shigella ; XLD : Xylose Lysine

Desoxycholate ; MKTT Müller Kauffmann TétraThionate ; nr : non réalisé.

* Etude ayant utilisée simultanément deux techniques d'enrichissement.

! Etude ayant utilisée des normes de références Afnor.

$¥$ Etude ayant utilisée des normes de références ISO.

Le tableau 1 présente les techniques utilisées pour l'isolement des Salmonella. Le pré-enrichissement a été rapporté dans dix-sept (17) études. Six (6) études ont utilisé simultanément deux techniques d'enrichissement (Chaiba et al., 2009; Ammar et al., 2010 ; Karou et al., 2013; Stevens et al., 2006 ; Abbassi-Ghozzi et al., 2012 ; Shilangale et al., 2012) et une seule étude a utilisé trois (3) techniques d'enrichissement (Kagambèga et al., 2010) . Cinq (5) études ont rapporté avoir utilisés des normes de références dont trois (3) références Afnor ((Bounar-Kechih et al., 2012); El Allaoui et al., 2014) ; Mezali et al., 2012) et deux (2) références ISO ((Karou et al., 2013) ; El Hussein et al., 2010). Les techniques et les méthodes d'isolement sont très variées.

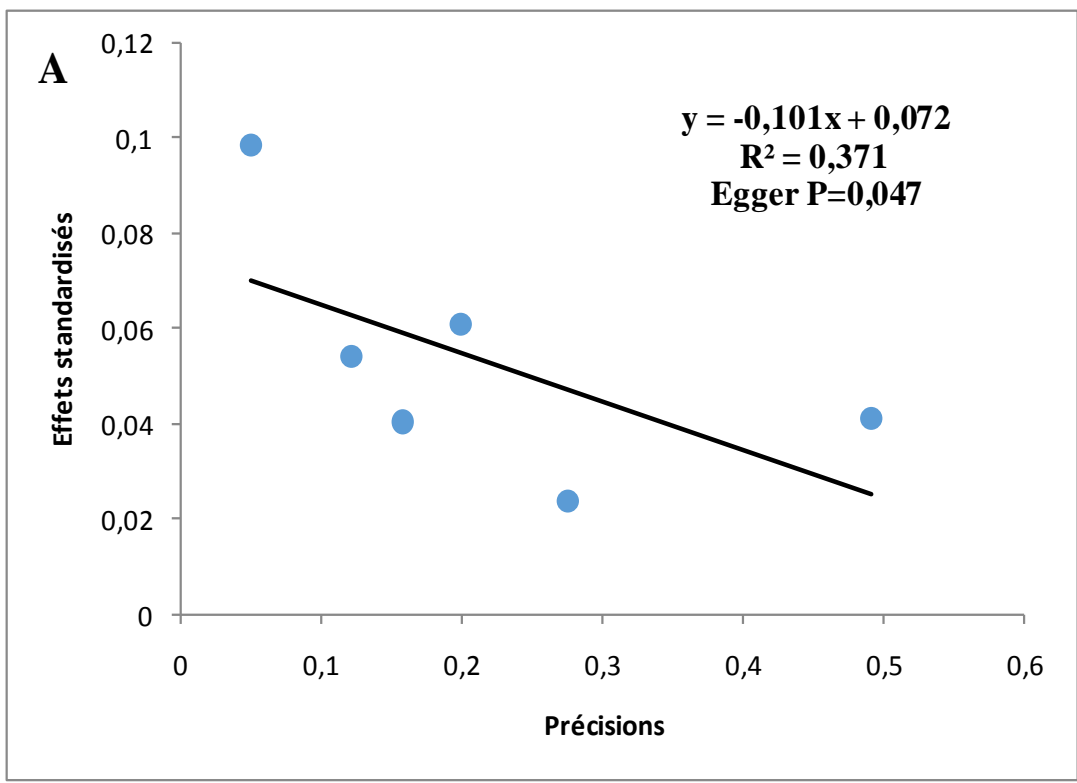




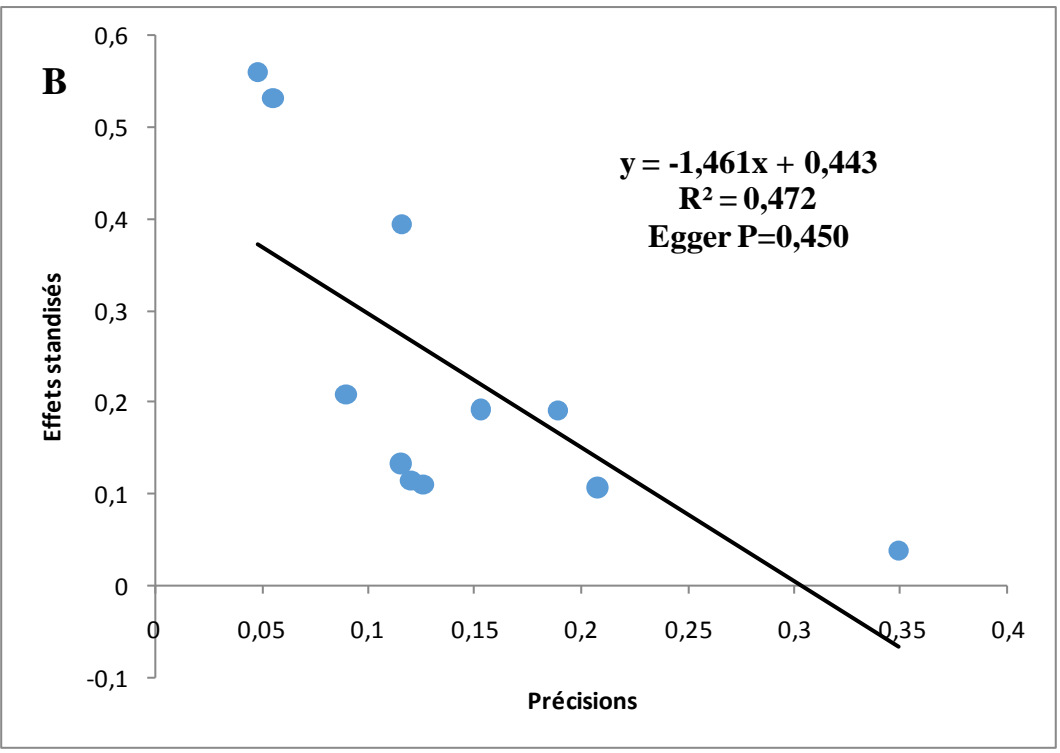

Figure 3 : Les courbes de régression (régression d'Egger) entre la précision des études (Variable indépendante) et l'effet standardisé (variable dépendante) des prévalences de Salmonella chez les humains (A), la volaille (B), le bétail(C) et les végétaux (D).

L'analyse de régression d'Egger a signalé l'existence de biais de publication concernant les prévalences randomisées des Salmonella seulement chez l'homme (intercept $=0,072 ; \mathrm{R}^{2}=0,371 ; \mathrm{p}=0,047$ ). Il n'y avait pas de biais de publication lorsque les prévalences ont été randomisées chez les végétaux (intercept $=-0,151$, $\mathrm{R}^{2}=0,590 ; \mathrm{p}=0,827$ ), chez les volaille (intercept $=0,443 ; \mathrm{R} 2=0,472 \mathrm{p}=0,450$ ) et chez le bétail (intercept $=0,646 ; \mathrm{R} 2=0,708 \mathrm{p}=0,064$ ).

\section{Source d'hétérogénéité}

Tableau 2 : Prévalences estimées et évaluation de l'hétérogénéité des Salmonella isolées chez les différents hôtes.

\begin{tabular}{|c|c|c|c|c|c|c|c|c|}
\hline & \multicolumn{4}{|c|}{ Données non transformées } & \multicolumn{4}{|c|}{ Données transformées } \\
\hline & $\begin{array}{l}\text { Prévalence \% } \\
\text { (IC95\%) }\end{array}$ & $\mathbf{Q}$ & $\mathbf{I}^{2}$ & $\begin{array}{c}\text { p- } \\
\text { value }\end{array}$ & $\begin{array}{l}\text { Prévalence \% } \\
\text { (IC95\%) }\end{array}$ & $\mathbf{Q}$ & $\mathbf{I}^{2}$ & $\begin{array}{c}\text { p- } \\
\text { value }\end{array}$ \\
\hline $\begin{array}{c}\text { Humai } \\
\mathbf{n}\end{array}$ & $5,21[3,37-7,06]$ & $\begin{array}{c}102 \\
04\end{array}$ & 95 & 0,016 & $5,3[4,8-5,9]$ & 3,03 & 0 & - \\
\hline $\begin{array}{c}\text { Volaill } \\
\text { e }\end{array}$ & $\begin{array}{c}22,34[17,04- \\
27,63]\end{array}$ & $\begin{array}{c}346 \\
41\end{array}$ & $\begin{array}{c}96,8 \\
2\end{array}$ & $\begin{array}{c}0,000 \\
2\end{array}$ & $23,51[18,03-28,98]$ & $\begin{array}{c}24,4 \\
8\end{array}$ & $\begin{array}{c}55,0 \\
8\end{array}$ & 0,004 \\
\hline Bétail & $\begin{array}{c}28,23[21,38- \\
35,07]\end{array}$ & $\begin{array}{c}295 \\
86\end{array}$ & $\begin{array}{c}97,6 \\
3\end{array}$ & 0,018 & $31,18[23,9-38,46]$ & 9,09 & $\begin{array}{c}22,9 \\
7\end{array}$ & 0,064 \\
\hline $\begin{array}{l}\text { Végéta } \\
\text { ux }\end{array}$ & $\begin{array}{c}23,93[22,61- \\
25,24]\end{array}$ & $\begin{array}{c}38,5 \\
4\end{array}$ & 0,92 & 0,127 & $24,79[21,36-28,23]$ & $\begin{array}{c}38,5 \\
4\end{array}$ & 0,92 & - \\
\hline
\end{tabular}


les données concernant les prévalences chez l'homme, la volaille et le bétail sont hétérogènes selon le test de $\mathrm{Q}$ et $\mathrm{I}^{2}$. Neanmoins, elles ont été marginalement hétérogènes chez les végétaux $\left(\mathrm{Q}=38,54 ; \mathrm{I}^{2}=0,92\right.$ et $\mathrm{p}=0,127)$. Lorsque les Prévalence ont été analysées dans un modèle aléatoire, les valeurs de $\mathrm{I}^{2}$ ont montré une hétérogénéité faible chez le bétail et modérée chez la volaille avec respectivement $\mathrm{I}^{2}=22,97 \%$ et $\mathrm{I}^{2}=55,08 \%$ ( Tableau 2). De ce fait, chez la volaille, les études ont été divisées en sousgoupes (groupes: d'Afrique du Nord, d'Afrique de l'Ouest et d'Afrique Australe). L'hétérogénéité s'est située dans le sous-groupe d'Afrique du nord. Elle est due au faible nombre des échantillons positifs trouvés par Ammar (Ammar et al., 2010) et la grande taille des échantillons positifs trouvés par Bounar-Kechih (Bounar-Kechih, 2012).

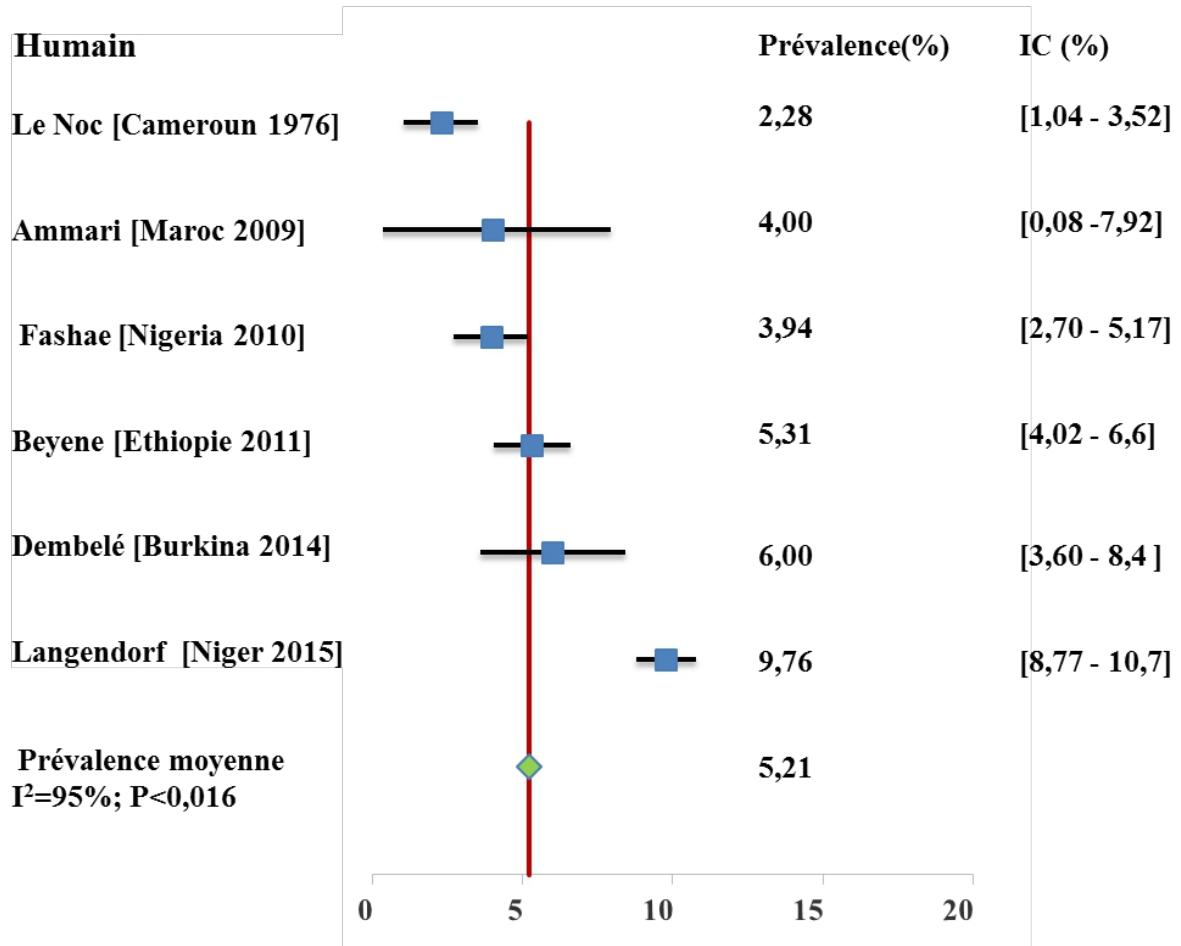

Figure 4 : Forests plots des prévalences non transformées des Salmonella isolées chez l'homme. Chaque ligne représente une étude. La ligne rouge centrale represente la tendance centrale. Les carrés centraux en bleus representent les prévalences des Salmonella de chaque étude. Les lignes en noires representent les intervalles de confiances. Le losange en vert représente la prévalence moyenne. 
Volaille

Chaiba [Maroc 2009]

Uche Orji [Nigeria 2010]

Ammar [Algérie 2010]

El Hussein [Soudan 2010]

Mezali [Algérie 2012]

Bounar- Kechih [Algérie 2012]

Ashraf [Egypte 2012]

Boko [Bénin 2012]

Karou [Côte d'Ivoire 2013]

Kagambèga [Burkina Faso 2013]

El Allaoui [Maroc 2014]

Prévalence moyenne $I^{2}=96,82 \% ; P<0,0001$

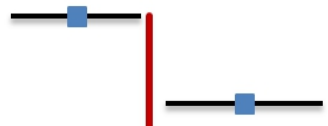

2,74

18,13

17,97

19,76

9,55

12,18

52,00

$\{48,13-55,87]$

54,86

$[37,27-72,45]$

10,33

[2,17 - 18,49]

22,34

$\begin{array}{rrrrrr}-10 & 10 & 30 & 50 & 70 & 90\end{array}$

$[-1,18-20,97]$

$[25,04-51,63]$

$[0,17-5,31]$

$[16,24-20,03]$

$[15,80-20,13]$

$[13,75-25,77]$

$[2,20-16,89]$

$[3,94-20,42]$

Figure 5 : Forests plots des prévalences non transformées des Salmonella isolées chez la volaille. Chaque ligne représente une étude. La ligne rouge centrale represente la tendance centrale . Les carrés centraux en bleus representent les prévalences des Salmonella de chaque étude. Les lignes en noires representent les intervalles de confiances. Le losange en vert représente la prévalence moyenne. 


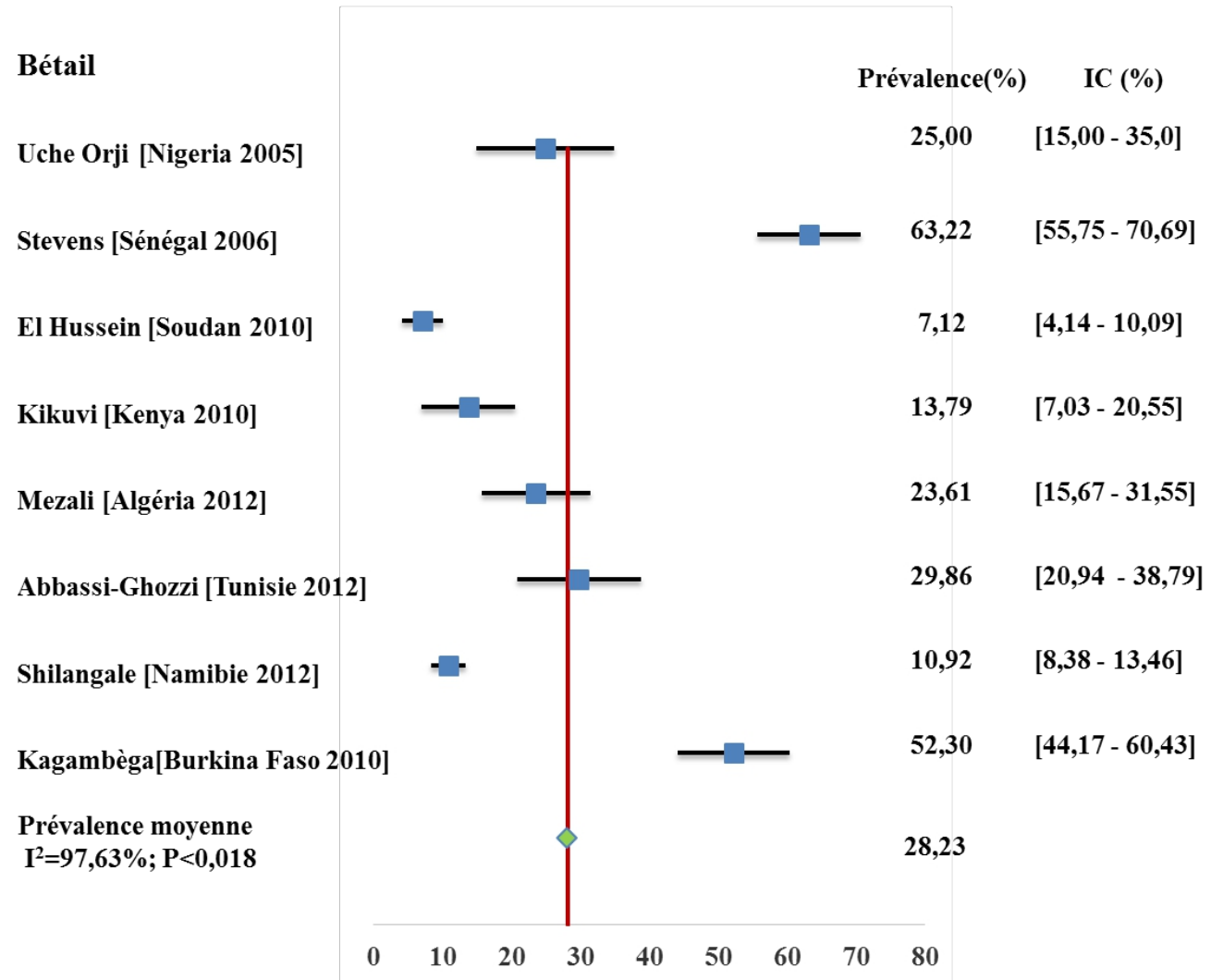

Figure 6 : Forests plots des prévalences non transformées des Salmonella isolées chez le bétail.

Chaque ligne représente une étude. La ligne rouge centrale represente la tendance centrale. Les carrés centraux en bleus representent les prévalences des Salmonella de chaque étude. Les lignes en noires representent les intervalles de confiances. Le losange en vert représente la prévalence moyenne. 


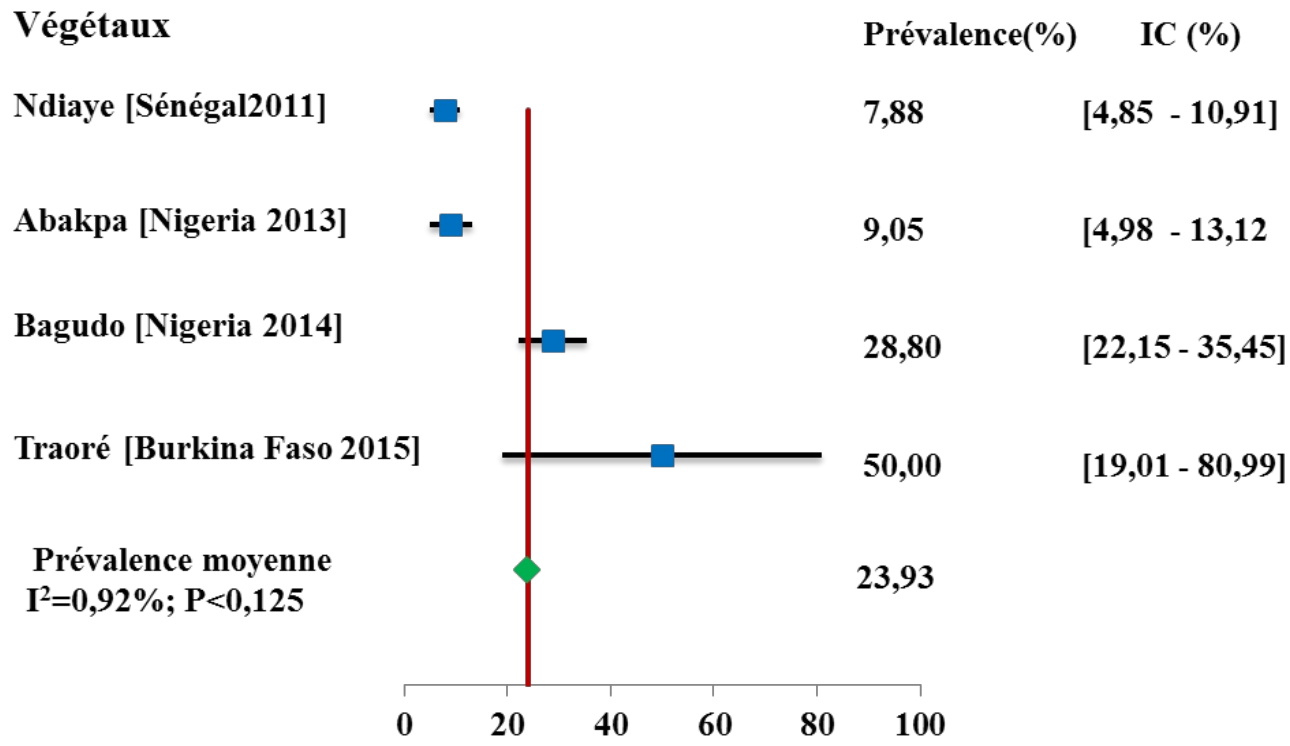

Figure 7 : Forests plots des prévalences non transformées des Salmonella isolées chez les végétaux.

Chaque ligne représente une étude. La ligne rouge centrale represente la tendance centrale. Les carrés centraux en bleus representent les prévalences des Salmonella de chaque étude. Les lignes en noires representent les intervalles de confiances. Le losange en vert représente la prévalence moyenne.

Tableau3 : Comparaison des différentes prévalences des Salmonella trouvées chez différents hôtes.

\begin{tabular}{|c|c|c|c|c|}
\hline Contrastes & $\begin{array}{l}\text { Différences de } \\
\text { prévalences }\end{array}$ & $95 \% \mathrm{IC}$ & $\mathrm{X} 2$ & $\begin{array}{c}\mathrm{P}- \\
\text { value }\end{array}$ \\
\hline $\begin{array}{c}\mathrm{p} \text { (Totale Humain) }-\mathrm{p} \text { (Totale } \\
\text { volaille) }\end{array}$ & $-0,13$ & $\begin{array}{c}-0,14 ;- \\
0,11\end{array}$ & $\begin{array}{c}- \\
17,77\end{array}$ & 0,0001 \\
\hline $\mathrm{p}$ (Totale Humain) - p (Totale bétail) & $-0,22$ & $\begin{array}{c}-0,24 ;- \\
0,20\end{array}$ & 21,62 & 0,0001 \\
\hline $\begin{array}{c}\mathrm{p}(\text { Totale Humain })-\mathrm{p} \text { (Totale } \\
\text { végétaux })\end{array}$ & $-0,08$ & $\begin{array}{c}-0,11 ;- \\
0,06\end{array}$ & $-6,39$ & 0,0001 \\
\hline $\mathrm{p}($ Totale volaille $)-\mathrm{p}$ (Totale bétail) & $-0,09$ & $\begin{array}{c}-0,12 ;- \\
0,07\end{array}$ & $-7,96$ & 0,0001 \\
\hline $\begin{array}{c}\mathrm{p}(\text { Totale volaille })-\mathrm{p} \text { (Totale } \\
\text { végétaux })\end{array}$ & 0,04 & $\begin{array}{c}0,015 \\
0,07\end{array}$ & 3,03 & 0,002 \\
\hline $\begin{array}{c}\mathrm{p} \text { (Totale bétail })-\mathrm{p} \text { (Totale } \\
\text { végétaux })\end{array}$ & 0,14 & 0,$10 ; 0,16$ & 8,49 & 0,0001 \\
\hline
\end{tabular}

La prévalence moyenne des Salmonella chez le bétail est supérieure à celle trouvée chez les autres hôtes. De plus, la prévalence trouvée chez l'homme est la plus faible. Pour ce qui est de la volaille, la prévalence des 
Salmonella trouvée est supérieure à celle trouvée chez les végétaux mais avec une faible différence ( $\mathrm{p}$-value $=0,002)$.

\section{Les sérotypes dominants}

Tableau 4 : Fréquence des dix sérotypes les plus prédominants isolés chez les différents hôtes

\begin{tabular}{|c|c|c|c|c|c|c|c|}
\hline Hôtes & Sérotypes & $\begin{array}{c}\text { Nombre } \\
\mathrm{s}\end{array}$ & $\begin{array}{c}\text { Prévalence } \\
\mathrm{s}\end{array}$ & Hôtes & Sérotypes & $\begin{array}{c}\text { Nombre } \\
\mathrm{s}\end{array}$ & $\begin{array}{c}\text { Prévalence } \\
\text { s }\end{array}$ \\
\hline \multirow{14}{*}{$\begin{array}{c}\text { Humai } \\
\mathrm{n}\end{array}$} & Typhimuriu & & & \multirow{14}{*}{ Bétail } & & & \\
\hline & $\mathrm{m}$ & 96 & $17,88 \%$ & & $\begin{array}{c}\text { Bredeney } \\
\text { Typhimuriu }\end{array}$ & 76 & $15,42 \%$ \\
\hline & Concord & 85 & $15,83 \%$ & & $\mathrm{~m}$ & 31 & $6,29 \%$ \\
\hline & Enteritidis & 73 & $13,59 \%$ & & Drac & 26 & $5,27 \%$ \\
\hline & Corvallis & 46 & $8,57 \%$ & & Muenster & 24 & $4,87 \%$ \\
\hline & Johannesbur & & & & & & \\
\hline & $\mathrm{g}$ & 15 & $2,79 \%$ & & Typhi & 24 & $4,87 \%$ \\
\hline & Maastricht & 12 & $2,23 \%$ & & Kentucky & 24 & $4,87 \%$ \\
\hline & Typhi & 12 & $2,23 \%$ & & Waycross & 18 & $3,65 \%$ \\
\hline & Banana & 11 & $2,05 \%$ & & Muenchen & 17 & $3,45 \%$ \\
\hline & Poona & 8 & $1,49 \%$ & & Corvallis & 15 & $3,04 \%$ \\
\hline & Dublin & 8 & $1,49 \%$ & & Chester & 14 & $2,84 \%$ \\
\hline & Autres & 171 & $31,84 \%$ & & Autres & 224 & $45,44 \%$ \\
\hline & Total & 537 & $100 \%$ & & Total & 493 & $100 \%$ \\
\hline \multirow{12}{*}{$\begin{array}{l}\text { Volaill } \\
\mathrm{e}\end{array}$} & Hadar & 106 & $13,54 \%$ & \multirow{12}{*}{$\begin{array}{l}\text { Végétau } \\
\mathrm{x}\end{array}$} & Typhi & 23 & $18,40 \%$ \\
\hline & $\begin{array}{c}\text { Typhimuriu } \\
\text { m }\end{array}$ & 74 & $9,45 \%$ & & $\begin{array}{c}\text { Typhimuriu } \\
\mathrm{m}\end{array}$ & 19 & $15,20 \%$ \\
\hline & Enteritidis & 57 & $7,28 \%$ & & Enteritidis & 11 & $8,80 \%$ \\
\hline & Derby & 56 & $7,15 \%$ & & Paratyphi C & 10 & $8,00 \%$ \\
\hline & Muenster & 37 & $4,73 \%$ & & Paratyphi B & 9 & $7,20 \%$ \\
\hline & Heidelberg & 37 & $4,73 \%$ & & Derby & 7 & $5,60 \%$ \\
\hline & Chester & 32 & $4,09 \%$ & & Paratyphi A & 6 & $4,80 \%$ \\
\hline & Kentucky & 29 & $3,70 \%$ & & Newport & 5 & $4,00 \%$ \\
\hline & Darc & 27 & $3,45 \%$ & & Korlebu & 4 & $3,20 \%$ \\
\hline & Oakland & 27 & $3,45 \%$ & & Colindale & 4 & $3,20 \%$ \\
\hline & Autres & 301 & $38,44 \%$ & & Autres & 27 & $21,60 \%$ \\
\hline & Total & 783 & $100 \%$ & & Total & 125 & $100 \%$ \\
\hline
\end{tabular}

La prévalence des dix (10) sérotypes prédominants isolés chez l'homme, la volaille, le bétail et les végétaux est respectivement de $68,16 \%$; $61,56 \% ; 54,56 \%$ et $78,40 \%$.

Chez l'homme, l'analyse a donné 573 échantillons sérotypés avec 88 sérotypes circulants. Les dix (10) sérotypes prédominants retrouvés chez 
l'homme sont respectivement: $S$. Typhimurium 17,88\%; $S$. Concord $15,83 \% ; S$. Enteritidis 13,59\%; S. Corvallis $8,57 \% ; S$. Johannesburg $2,79 \%$; S. Maastricht 2,23\% ; S.Typhi 2,23\% ; S.Banana 2,05\% ; S. Poona $1,49 \%$; S.Dublin 1,49\%. En Afrique de l'Ouest, $S$. Typhimurium est le sérotype le plus prédominant avec une prévalence $20,91 \%$ suivi de $S$. Enteritidis $16,59 \%$ et de $S$. Corvallis $11,06 \%$.

Chez la volaille, il y a 783 échantillons sérotypés dont 48 sérotypes circulants. Les dix (10) sérotypes les plus fréquemment trouvés par ordre décroissant sont : $S$. Hadar 13,54\%; $S$. Typhimurium 9,45\%, $S$. Enteritidis $7,28 \%, S$. Derby 7,15\%, S. Muenster 4,73\%, S. Heidelberg 4,73\%, S. Chester 4,09\%, S. Kentucky 3,70\%, S. Drac 3,45\%, S. Oakland 3,45\%.

$S$. Hadar est le sérotype prédominant en Afrique de l'Ouest (AO) avec une prévalence de $22,85 \%$ suivi respectivement de $S$. Derby $12,67 \%$ et $S$. Muenster $8,37 \%$ par contre en Afrique du Nord est les sérotypes prédominants sont $S$. Typhimurium $20,64 \%, S$. Enteritidis $14,95 \%$ et $S$. Heidelberg 13,17\%.

$\mathrm{Au}$ niveau du bétail, 493 échantillons sont collectés avec 71 sérotypes circulants. Les dix (10) sérotypes les plus fréquemment trouvés par ordre décroissant sont: $S$. Bredeney $15,42 \% ; S$. Typhimurium 6,29\%; $S$. Drac 5,27\% ; $S$. Muenster 4,87\% ; $S$. Typhi 4,87\% ; $S$. Kentucky 4,87\% ; $S$. Waycross $3,65 \%$; $S$. Muenchen 3,45\%; S. Corvallis 3,04\%; S. Chester $2,84 \%$.

$S$. Bredeney est le sérotype prédominant en Afrique de l'Ouest (AO) avec une prévalence de $19,56 \%$ suivi de $S$. Muenster $10,47 \%$ et $S$. Drac $7,16 \%$.

Chez les végétaux, on trouve 125 échantillons sérotypés dont 29 sérotypes circulants. Les sérotypes trouvés sont respectivement $S$. Typhi $18,40 \%$; $S$. Typhimurium 15,20\%; S. Enteritidis $8,80 \%$; S. Paratyphi C $8,00 \% ; S$. Paratyphi B 7,20\%; $S$. Derby $5,60 \% ; S$. Paratyphi A 4,80\%; $S$. Newport $4,00 \%$. A ce niveau, toutes les études proviennent de l'Afrique de l'Ouest.

\section{Discussion}

La recherche par mots clés dans les moteurs de recherche a permis de ressortir cent trente-et-une (131) études. Plusieurs pays ont effectué des études sur les Salmonella mais la plupart sont des études rétrospectives. Ces études ont donc été exclues de l'analyse. D'autres études ont porté sur des sérotypes spécifiques ou sur des Salmonella non-typhoïdiques, elles ne sont donc pas retenues. Enfin seulement vingt-cinq (25) études présentant toutes les caractéristiques recherchées sont retenues pour être analysées (figure1). Ces études ont porté sur des Salmonella isolées chez l'homme, la volaille, le bétail et les végétaux. 
Les techniques utilisées pour l'isolement des Salmonella (tableau1) pourraient sous-estimer le nombre des échantillons positifs. En effet l'utilisation du pré-enrichissement augmente la sensibilité des cultures mais le nombre d'isolats pourrait différer selon les milieux utilisés (Hoorfar et al., 1988). Les souches de Salmonella peuvent présenter des caractéristiques différentes d'un milieu d'enrichissement à un autre et il pourrait y avoir une identification différentielle d'un échantillon à un autre (Singer et al., 2009). Le taux d'isolement et la sensibilité des Salmonella sont plus élevés lorsqu'on utilise un milieu d'enrichissement plutôt que la culture directe. De ce fait, les variations des estimations des prévalences attribuant une hétérogénéité pourrait être dues aux différentes populations étudies et aux méthodes microbiologiques utilisées pour détecter les Salmonella. Mais l'analyse de ces études sous forme de modèle aléatoire (tableau 2) a permis de réduire les hétérogénéités. Car le modèle d'étude aléatoire considère les études comme des échantillons d'une même étude. Les résultats montrent aussi une disparition du biais de publication chez la volaille, le bétail et les végétaux lorsque les prévalences ont été randomisées. Mais le biais de publication persiste chez l'homme. Plusieurs facteurs peuvent être à la base de ce biais de publication : les caractéristiques des populations évaluées par l'étude, les tests de diagnostics utilisés dans l'étude,...

La prévalence des Salmonella isolées chez l'homme est de 5,21\% (figure 4). Elle atteint 9,76\% dans une étude réalisée au Niger en 2015 (référence). Par contre, elle est de 22,34\%,28,23\% et 23,93\% respectivement chez la volaille, le bétail et les végétaux (figures 5,6 et 7). Les prévalences élevées des Salmonella chez la volaille, le bétail et les végétaux comparés à la prévalence chez l'homme montrent que les Salmonella sont très présentes dans l'environnement. En effet, il a été rapporté que Salmonella un agent pathogène persistant dans l'environnement. Elle est capable de survivre et de proliférer dans divers environnements d'où elle contamine l'homme (Winfield et al., 2003).

Le tableau 4 décrit la prédominance des sérotypes dans différents hôtes. Chez l'homme, le sérotypes prédominant est $S$. Typhimurium. Il a été rapporté dans trois études (Le Noc et al., 1976 ; Dembélé et al., 2014 ; Langendorf et al., 2015).

Chez la volaille, le sérotype prédominant est $S$. Hadar. Cependant les études réalisées en Afrique du Nord $S$. Typhimurium en première position (Chaiba et al., 2009; Ammar et al., 2010). Chez le bétail, le sérotype prédominant est $S$. Bredeney mais une seule étude l'a décrit comme sérotype prédominant (Antoine et al., 2003). Parmi les dix (10) sérotypes prédominants, un seul est présent chez les quatre (4) hôtes, il s'agit $S$. Typhimurium. Deux (2) sérotypes sont présents chez trois (3) hôtes différents : $S$. Enteritidis chez l'homme, la volaille, les végétaux et $S$. Typhi 
chez l'homme, le bétail, les végétaux. Quatre (4) sérotypes sont présents chez deux (2) hôtes différents : $S$. Corvallis (chez l'homme et le bétail), $S$. Drac (, $S$. Derby, $S$. Chester (tableau 4).

Les différents résultats montrent une large distribution et une grande diversité des sérotypes à travers différents hôtes et différentes régions. La prédominance des sérotypes diffère d'un continent à un autre. En Europe en $2014, S$. Enteritidis $(44,4 \%)$ et $S$. Typhimurium $(17,4 \%)$ ont été les sérotypes prédominants isolés chez l'homme. Chez la volaille, le sérotype le plus rapporté est $S$. Infantis $(38,3 \%)$ suivi de $S$. Mbandaka $(12,1 \%)$ et $S$. Enteritidis (11,9\%). Chez le bétail, le sérotype prédominant est $S$. Typhimurium (46,8\%) suivi de $S$. Dublin (31,3\%) (The European Union summary report 2014). Aux USA, les trois (3) sérotypes prédominants chez l'homme sont: $S$. Enteritidis (15.1\%) et $S$. Typhimurium (12,8\%) et $S$. $\begin{array}{llll}\text { Newport } & (8,3 \%) & \text { (CDC }\end{array}$ (http://www.cdc.gov/salmonella/outbreaks.html). Des éclosions de Salmonella suite au contact avec des volailles ont été rapportées. Les sérotypes les plus décrits sont $S$. Typhimurium, $S$. Kentucky, et $\mathrm{S}$. Heidelberg. Chez le bétail, le sérotype prédominant est $S$. Dublin $(31.8 \%)$ suivi du sérotype $S$. Montevideo $(10.9 \%)$ et de $S$. Cerro (6.6\%)

La fluctuation des sérotypes par hôtes pourrait être due aux différences dans les interactions hôtes-sérotypes. Les caractéristiques génétiques des hôtes peuvent affecter les Salmonella. En outre, un sérotype peut avoir des capacités différentes pour infecter des hôtes différents. De plus les facteurs environnementaux peuvent influencer la survie des sérotypes et leur distribution entre les différents hôtes.

\section{Conclusion}

Cette étude permet une meilleure compréhension de la distribution et de la prévalence des Salmonella en Afrique. La prévalence des Salmonella est élevée quel que soit 1'hôte. Le germe Salmonella présente une grande diversité d'un hôte à l'autre. Les sérotypes majeurs retrouvés chez l'homme en Afrique sont $S$. Typhimurium, $S$. Enteritidis et $S$. Typhi. Chez la volaille, le sérotype prédominant est $S$. Hadar par contre en Afrique du Nord, c'est $S$. Typhimurium qui prédomine. Cette étude peut être une base de référence pour des recherches épidemiologiques de la diversité des Salmonella dans les pays africains.

\section{References:}

1. Abbassi-Ghozzi A., Jaouani , Aissa R.B., Martinez-Urtaza J. (2012) :Antimicrobial resistance and molecular analysis of nontyphoidal Salmonella isolates from human in Tunisia. Pathologie 
Biologie

Volume59,n4pages 207-212. Doi : 10.1016/j.patbio.2010.06.001.

2. Abdellah E, A. , Fouzia R. Fi., Najiya A., Ilham N., Bouchra O., Aboulkacem, Adil E., Derouich A, Bouchrif B (2014) : Prevalence, Antibio-Resistance and Risk Factors for Salmonella inBroiler Turkey Farms in the Province of Khémisset (Morocco):J. World's Poult. Res. 4(1): 20-29

3. Abakpaa, G.O., Umoha V.J., Ameha J.B., Yakubua S.E., Kwaga J.K.P., S. Kamaruzamanc (2013) : Diversity and antimicrobial resistance of Salmonella enterica isolated from fresh produce and environmental samples. Environmental Nanotechnology, Monitoring \& Management 3 38-46

4. El Hussein., Elmadiena, M. M. Nor, Elsaid.S.M.; Siddig.M. A.M., Muckle.C.A., Cole L., Wilkie E., Mistry K., (2010) : Prevalence of Salmonella enterica subspecies enterica Serovars in Khartoum State, Sudan,Research Journal of Microbiology; Vol. 5 Issue 10, p966.

5. Ammar A., Nadir A., Bennoune O., Kassah-laouar A.,(2010): Survey of Salmonella serovars in broilers and laying breeding reproducers in Eastern Algeria. J Infect Dev Ctries 2010; 4(2):103-106.

6. Ammari S., Laglaoui A., En-Nanei L., Bertrand S., Wildemauwe C., Barrijal S., Abid M.(2009) : Isolation, drug resistance and molecular characterization of Salmonella isolates in northern Morocco.J Infect Dev Ctries;3(1):41-9.

7. Ashraf M.A., Tadashi S.,(2012): Genetic analysis of multiple antimicrobial resistance in Salmonella isolated from diseased broilers in Egypt. Microbiol Immunol; 56: 254-261 doi:10.1111/j.13480421.2012.00429.x

8. Bagudo A. I., Tambuwal F. M., Faleke O. O., Egwu O. O.and Aliero A. A et al., (2014): Prevalence of salmonella serotypes in Sokoto abattoir effluents and vegetables cultivated around the abattoir Microbiology Research International Vol. 2(2), pp. 13-17, April 2014 ISSN: 2354-2128.

9. Beyene G., Nair S., Asrat D., Mengistu Y., Engers H., Wain J.(2011) : Multidrug resistant Salmonella Concord is a major cause of salmonellosis in children in Ethiopia.J Infect Dev Ctries;5(1):23-33.

10. Boko CK, Kpodekon TM, Duprez JN, Imberechts H, Taminiau B, Bertrand S, Mainil JG. (2013) : Identification and typing of Salmonella enterica serotypes isolated from guinea fowl (Numida meleagris) farms in Benin during four laying seasons (2007 to 2010).Avian Pathol.42(1):1-8.

11. Bounar-Kechih S, Hamdi TM, Mezali L, Assaous F, Rahal K. (2012): Antimicrobial resistance of 100 Salmonella strains isolated from 
Gallus gallus in 4 wilayas of Algeria.Poult Sci. 2012 May;91(5):1179-85.

12. Bounar-Kechih S, Hamdi TM, Mezali L, Assaous F, Rahal K. (2012): Antimicrobial resistance of 100 Salmonella strains isolated from Gallus gallus in 4 wilayas of Algeria.Poult Sci. 2012 May;91(5):1179-85.

13. CDC: Reports of selected Salmonella outbreak investigations. Atlanta, GA: $\quad$ CDC; 2016 [http://www.cdc.gov/salmonella/outbreaks.html].

14. Chaiba Abdellah, Rhazi Filali Fouzia, Chahlaoui Abdelkader, Soulaymani Bencheikh ( 2009 ) : Prevalence and anti-microbial susceptibility of Salmonella isolates from chicken carcasses and giblets in Meknès, Morocco,African Journal of Microbiology Research Vol. 3(5) pp. 215-219.

15. Coulibaly K.J., S. Bakayoko, K.E. Coulibaly, G.T. Karou, G.B. Goualie, L. Akesse, C. Gbonon, C. Boni-cisse, K.S. Koffi, E. Ekaza, A. N'Douba et M. Dosso (2010) : Biodiversité des Salmonella à Abidjan : Etude des isolats de 2003 à 2009 par le centre de référence de l'Institut Pasteur ; Revue Africaine de Santé et de Productions Animales E.I.S.M.V. de Dakar.pp 19-23.

16. Dembélé R., Isidore J.O., Kagambèga., Kiessoun K., Bagré S et al (2014) : Serotyping and antimicrobial susceptibility of Salmonella isolated from children under five years of age with diarrhea in rural Burkina Faso, Afr. J. Microbiol. Res.Vol.8(34), pp. 3157-3163.

17. Abdellah El A., Fouzia R., Nassri N et al. ( 2014): Prevalence, Antibio-Resistance and Risk Factors for Salmonella in Broiler Turkey Farms in the Province of Khémisset (Morocco) . Journal of World's Poultry Research

18. Fashae k Fashae, K., Ogunsola, F., Aarestrup, F. M., Hendriksen, R. S (2010) : Antimicrobial susceptibility and serovars of Salmonella from chickens and humans in Ibadan, Nigeria. J Infect Dev Ctries; 4(8):pp 484-494.

19. Global Foodborne Infections Network

$(\mathrm{GFN})$ http://apps.who.int/salmsurv/en/

20. Hoorfar D, Baggesen DL (1988): Importance of pre-enrichment media for isolation of Salmonella spp. from swine and poultry. FEMS Microbiol Lett, 169:125-130.

21. Kagambèga A.L., Taru L., Aulu L., Traoré A. S., Barro N., Siitonen A., Haukka K., ( 2013) : Prevalence and characterization of Salmonella enterica from the feces of cattle, poultry, swine and hedgehogs in Burkina Faso and their comparison to human 
Salmonella isolates. BMC Microbiology, 13:253 http://www.biomedcentral.com/1471-2180/13/253.

22. Karou G T, Honoré Ouattara, Souleymane Bakayoko (2013) : Prevalence of Salmonella and distribution of Serovars Isolated from Retail RawChicken Gizzards in Abidjan, Côte D'ivoire; Octa. J. Biosci. Vol. 1(2): 115-121.

23. Kikuvi G, Jackson N. Oi, Eric S. M et al. (2010) : Serotypes and antimicrobial resistance profiles of Salmonella isolates from pigs at slaughter in Kenya. J Infect Dev Ctries 2010; 4(4):243-248.

24. Langendorf C, Le Hello S, Moumouni A, Gouali M, Mamaty A-A, Grais RF, et al. (2015) : Enteric Bacterial Pathogens in Children with Diarrhea in Niger: Diversity and Antimicrobial Resistance. PLoS ONE 10(3): e0120275. doi:10.1371/journal.

25. Le Noc P et D Le Noc - (1976) : le portage sain des Salmonella en milieu africain: enquete chez des ecoliers de Cote d'Ivoire. et du Cameroun. Ann. Soc. Belge Méd. trop.56, 2, 65-72

26. Mezali .L ( 2012 ) : Prevalence and Antimicrobial Resistance of Salmonella Isolated from Meat and Meat Products in Algiers. Foodborne pathogens and disease.Vol 9, $\mathrm{N}^{\circ} 6 . \quad$ DOI: 10.1089/fpd.2011.1032.

27. Michel Gedda (2015) Traduction française des lignes directrices PRISMA pour l'écriture et la lecture des revues systématiques et des méta-analyses. Kinesither Rev 2015;15(157):39-44.

28. Uche Orji.M , Henry C. Onuigbo, Theodore I. Mbata (2005) : Isolation of Salmonella from poultry droppings and other environmental sources in Awka, Nigeria, International Journal of Infectious Diseases 9, 86-89.

29. Ndiaye M.L. , Dieng Y., Nian S.g, H.R. Pfeifer, M. Tonolla, R. Peduzzi (2011) : Effect of irrigation water on the incidence of Salmonella spp. on lettuce produced bu urban agriculture and sold in markets in Dakar Senegal. Afr. J. Microbiol. Res., 5 (19) (2011), pp. 2885-2890

30. Nicholas A Feasey FRCPath a b, Gordon Dougan PhD c, Robert A Kingsley $\mathrm{PhD}$ c, Robert $\mathrm{S}$ Heyderman $\mathrm{PhD}$ a d, Dr Melita A Gordon MD ( 2012) :Invasive non-typhoidal Salmonella disease: an emerging and neglected tropical disease in Africa,The Lancet, Volume 379, Issue 9835, pp 2489 - 2499.

31. René Hendriksen PhD Thesis January 2010: Global epidemiology of non-typhoidal Salmonella infections in humans.

32. Shannon E. Majowicz, Jennie Musto, Elaine Scallan, Frederick J. Angulo, Martyn Kirk, Sarah J. O'Brien, Timothy F. Jones, Aamir Fazil, and Robert M. Hoekstra (2010) : for the International 
Collaboration on Enteric Disease 'Burden of Illness' Studies: The Global Burden of Nontyphoidal Salmonella Gastroenteritis Clinical Infectious Diseases; 50:pp 882-889.

33. Shilangale R.P, Di Giannatale E, Chimwamurombe PM, Kaaya GP. (2012): Prevalence and antimicrobial resistance pattern of Salmonella in animal feed produced in Namibia. Vet Ital;48(2):125-32.

34. Singer R.S, Mayer A.E, Hanson T.E, Isaacson R.E (2009): Do microbial interactions and cultivation media decrease the accuracy of Salmonella surveillance systems and outbreak investigations? J Food Prot, 72:pp707-713.

35. Stevens A., Kaboré Y, Perrier-Gros-Claude, Millemann Y, Brisabois A, et al (2006): Prevalence and antibiotic-resistance of Salmonella isolated from beef sampled from the slaughterhouse and from retailers in Dakar (Senegal) International Journal of Food Microbiology, Volume 110, Issue 2, 15 July 2006, Pages 178-186

36. Tadesse G., (2014a): Prevalence of human Salmonellosis in Ethiopia : a systematic review and meta-analysis. BMC Infectious Diseases 2014, 14:pp88. doi:10.1186/1471- 2334-14-88.

37. Tadesse G., Tesfaye SA (2014b) : meta-analysis of the prevalence of Salmonella in food animals in Ethiopia. BMC Microbiology, 14:270 http://www.biomedcentral.com/1471-2180/14/270. doi:10.1186/s12866-014-0270-y

38. Traoré et al. (2015) : Prevalence and diversity of Salmonella enterica in water, fish and lettuce in Ouagadougou, Burkina Faso BMC Microbiology 15:151.

39. Tennant SM, Diallo S, Levy H, Livio S, Sow SO, Tapia M, Fields PI, Mikoleit M, Tamboura B, Kotloff KL, Nataro JP, Galen JE, Levine MM (2010) : Identification by PCR of non-typhoidal Salmonella enterica serovars associated with invasive infections among febrile patients in Mali.PLoS Negl Trop Dis.4(3):e621

40. The European Union summary report on trends and sources of zoonoses, zoonotic agents and food-borne outbreaks in 2014 EFSA (2015): 13(12):4329.

41. Wadula J., Von Gottberg A., Kilner D., De Jong G., Cohen C., et al (2006) :: Nosocomial outbreak of extended-spectrum beta-lactamaseproducing Salmonella isangi in pediatric wards; The Pediatric infectious disease journal. 25:843-4

42. Winfield, M.D. and Groisman, E.A. (2003). Role of non-host environments in the lifestyles of Salmonella and Escherichia coli. Appl. Environ. Microb., 69: 3687-3694. 